See discussions, stats, and author profiles for this publication at: https://www.researchgate.net/publication/274711936

\title{
Flavonoids from Eupatorium illitum and Their Antiproliferative Activities
}

Article in Pharmacognosy Journal · May 2015

CITATIONS

4

10 authors, including:

8

Quírico Castillo

Universidad Autónoma de Santo Domingo

7 PUBLICATIONS 33 CITATIONS

SEE PROFILE

José M Padrón

Universidad de La Laguna

223 PUBLICATIONS 2,005 CITATIONS

SEE PROFILE

Some of the authors of this publication are also working on these related projects:

American Foulbrood View project

Ruthenium complexes as anticancer drugs View project
READS

112

José Luis Eiroa

Universidad de Las Palmas de Gran Canaria

32 PUBLICATIONS 373 CITATIONS

SEE PROFILE

Ernesto Abel-Santos

University of Nevada, Las Vegas

66 PUBLICATIONS 1,555 CITATIONS

SEE PROFILE 


\title{
Flavonoids from Eupatorium illitum and Their Antiproliferative Activities
}

\author{
Quírico A Castillo*1,2, Jorge Triana ${ }^{3}$, José L Eiroa ${ }^{3}$, José M Padrón ${ }^{4}$, Gabriela B Plata4, \\ Ernesto V Abel-Santos ${ }^{5}$, Luis A. Báez¹, Diana C Rodríguez¹, Marco A Jiménez², \\ María F Pérez-Pujols ${ }^{1}$
}

${ }^{1}$ Escuela de Química, Facultad de Ciencias, Universidad Autónoma de Santo Domingo, Ciudad Universitaria, Santo Domingo D.N., Dominican Republic.

${ }^{2}$ Instituto de Química, Facultad de Ciencias, Universidad Autónoma de Santo Domingo, Ciudad Universitaria, Santo Domingo D.N., Dominican Republic.

${ }^{3}$ Departamento de Química, Unidad Asociada al CSIC, Universidad de Las Palmas de Gran Canaria, Campus de Tafira, 35017 Las Palmas de Gran Canaria, Canary Islands, Spain.

${ }^{4}$ BioLab, Instituto Universitario de Bio-Orgánica "Antonio González" (IUBO-AG), Centro de Investigaciones Biomédicas de Canarias (CIBICAN), C/Astrofísico Francisco Sánchez 2, 38206. La Laguna, Spain.

${ }^{5}$ Department of Chemistry and Biochemistry, University of Nevada-Las Vegas, Las Vegas, NV 89154.

\begin{abstract}
Objective: To isolate the chemical constituents of the aerial parts from Eupatorium illitum, elucidate their structures and evaluate their antiproliferative activity on human cancer cell lines. Materials and Methods: The ethanolic extract of Eupatorium illitum afforded five compounds, which were characterized using spectroscopic techniques and by comparison with data from the literature. Antiproliferative activities of selected isolates were evaluated. Results: The flavonoids Kumatakenin (1), Ermanin (2), 7-methoxy-aromadendrin (3) and Naringenin (4), together with 4-hydroxybenzoic acid (5) were isolated. Compounds 1, 2, 4 and 5 were evaluated for their antiproliferative activity on the human cancer cell lines A549 (lung), HBL-100 (breast), HeLa (cervix), SW1573 (lung), and T-47D (breast) presenting a wide range of bioactivities. In general, best results were observed for $\mathbf{5}$. Conclusion: Compounds 1-5 are reported for first time from Eupatorium illitum. Isolated phytochemicals show moderate to low antiproliferative activities when evaluated on the aforementioned human cancer cell lines.
\end{abstract}

Key words: Eupatorium illitum, Ermanin, 4-Hydroxybenzoic acid, Kumatakenin, 7-Methoxy-aromadendrin, Naringenin.

\section{INTRODUCTION}

Eupatorium illitum Urb. (Asteraceae) is an endemic plant from Dominican Republic. ${ }^{1}$ The genus Eupatorium comprises of nearly 1,200 species distributed mainly in the tropical regions of Americas, Europe, Africa and Asia ${ }^{2}$. Flavonoids isolated from species of this genus have been reported to

*Corresponding author:

Prof. Quírico A Castillo

Escuela de Química and Instituto de Química,

Facultad de Ciencias, Universidad Autónoma de Santo Domingo,

Ciudad Universitaria, Santo Domingo D.N.,

Dominican Republic.

Phone No: +809 5358273 ext. 4406

E-mail: qcastillo55@uasd.edu.do

DOI: $10.5530 / p j .2015 .3 .6$ show cytotoxic activity against cancer cell lines. ${ }^{3-5}$ In the present study, we report compounds 1-5, isolated for first time from E. illitum, and their antiproliferative activities on human cancer cell lines A549, HBL-100, SW1573, HeLa, and T-47D.

\section{MATERIALS AND METHODS}

\section{General}

IR spectra were recorded using a Hyperion 3000 Fourier transform infrared microscope. ${ }^{1} \mathrm{H}$ and ${ }^{13} \mathrm{C}$ NMR spectra were obtained on a Bruker Avance III spectrometer with cryoprobe operating at $700 \mathrm{MHz}$ in ${ }^{1} \mathrm{H}$ and $175 \mathrm{MHz}$ in 
${ }^{13} \mathrm{C}$ NMR respectively. DMSO- $\mathrm{d}_{6}$ was used as solvent. HRESIMS was performed with a Bruker Maxis 4G QTOF mass spectrometer; presented results are for negative ion mode. Silica gel 60 (particle sizes $0.040-0.063 \mathrm{~mm}$ and 0.015-0.040 mm, Merck KGaA, Darmstadt, Germany) was used for column chromatography. Analytical and preparative TLC was developed on Silica gel $60 \mathrm{~F}_{254}$ plates (Merck KGaA, Darmstadt, Germany).

\section{Plant Material}

The aerial parts of Eupatorium illitum Urb. (Asteraceae) were collected on June 2010 at Sierra de Bahoruco, Provincia Pedernales, Dominican Republic. The plant material was identified by Teodoro Clase, botanist at Jardín Botánico Nacional "Dr. Rafael Ma. Moscoso", Santo Domingo, Dominican Republic, where a voucher specimen (JBSD 121457) has been deposited.

\section{Extraction and Isolation}

Aerial parts of Eupatorium illitum were shade, air dried, and ground to a fine powder. The ground material (617 g) was exhaustively extracted with $95 \% \mathrm{EtOH}$ in a Soxhlet apparatus. The resulting crude extract $(90.9 \mathrm{~g})$ was dissolved in $95 \% \mathrm{EtOH}(1.2 \mathrm{~L})$ and treated with a $5 \%$ lead acetate solution (1L) to precipitate chlorophyll. After 24 hours, the mixture was filtered over paper, concentrated in vacuo to remove most of the EtOH, and extracted successively with hexane $(6 \times 750 \mathrm{~mL})$ and ethyl acetate $(6 \times 1 \mathrm{~L})$. The ethyl acetate residue $(10.7 \mathrm{~g})$ was washed with distilled water $(2 \times 1 \mathrm{~L})$, dried over anh. $\mathrm{Na}_{2} \mathrm{SO}_{4}$, and subjected to CC (Si gel 0.040-0.063 mm) using hexane-acetone mixtures of increasing polarity to afford 22 fractions (F1-F22). Fraction 15 afforded $\mathbf{1}(190.6 \mathrm{mg})$ as a yellow precipitate; supernatant F15 was eluted over a column of Si gel (0.015$0.040 \mathrm{~mm}$ ) with a gradient system of hexane-AcOEt (3:1 to pure AcOEt) affording 19 subfractions. Subfraction F15-13 gave 2 (9.2 $\mathrm{mg}$ ) after PTLC (Si gel) using a mixture of hexane-AcOEt (7:3).

Fraction 16 was chromatographed over a column of $\mathrm{Si}$ gel $(0.015-0.040 \mathrm{~mm})$ with a gradient system of hexaneAcOEt (7:3 to pure AcOEt), affording 16 subfractions. Sub fraction F16-15 was recromatographed over Si gel (0.015$0.040 \mathrm{~mm})$ using a gradient system of hexane-AcOEt $(7: 3$ to pure AcOEt) to yield 21 subfractions. PTLC (Si gel) of subfraction F16-15-15 using a mixture of hexane-AcOEt (6.5:3.5, twice) afforded $\mathbf{3}(5.8 \mathrm{mg})$ and 4 (5.6 mg).

Fraction 21 was chromatographed over a Si gel (0.015-0.040 $\mathrm{mm})$ column with a gradient system of hexane-acetone (6.5:3.5 to pure acetone) to yield $\mathbf{5}(13.9 \mathrm{mg})$.

\section{Antiproliferative assays}

Biology

All starting materials were commercially available researchgrade chemicals and used without further purification. Fetal calf serum (FCS) was purchased from Gibco (Grand Island, NY), trichloroacetic acid (TCA) and glutamine were from Merck (Darmstadt, Germany), and RPMI 1640 medium penicillin $G$, streptomycin, DMSO and sulforhodamine B (SRB) were from Sigma (St Louis, MO).

\section{Cell lines and culture}

The human solid tumor cell lines A549, HBL-100, HeLa, SW1573, and T-47D were used in this study. These cell lines were a kind gift from Prof. G. J. Peters (VU Medical Center, Amsterdam, The Netherlands). Cells were maintained in $25 \mathrm{~cm}^{2}$ culture flasks in RPMI 1640 supplemented with $5 \%$ heat inactivated fetal calf serum and $2 \mathrm{mM} \mathrm{L}$-glutamine in a $37^{\circ} \mathrm{C}, 5 \% \mathrm{CO}_{2}, 95 \%$ humidified air incubator. Exponentially growing cells were trypsinized and re-suspended in antibiotic containing medium (100 units penicillin $G$ and $0.1 \mathrm{mg}$ of streptomycin per $\mathrm{mL}$ ). Single cell suspensions were counted using Orflow's Moxi ${ }^{\mathrm{Z}}$ automated cell counter (Ketchum, ID) and dilutions were made to give the appropriate cell densities for inoculation onto 96-well microtiter plates. Cells were inoculated in a volume of $100 \mu \mathrm{L}$ per well at densities of 10000 (A549, HBL-100, HeLa and SW1573), and 15000 (T-47D) cells per well, based on their doubling times.

\section{Chemosensitivity testing}

Compounds were initially dissolved in DMSO at 400 times the desired final maximum test concentration. Control cells were exposed to an equivalent concentration of DMSO $(0.25 \% \mathrm{v} / \mathrm{v}$, negative control). Each agent was tested in triplicate at different dilutions in the range of $1-100 \mu \mathrm{M}$. The drug treatment was started on day 1 after plating. Drug incubation times were $48 \mathrm{~h}$, after which time cells were precipitated with $25 \mu \mathrm{L}$ ice-cold TCA $(50 \% \mathrm{w} / \mathrm{v})$ and fixed for 60 min at $4^{\circ} \mathrm{C}$. Then the SRB assay was performed. ${ }^{6}$ The optical density (OD) of each well was measured at 492 nm, using BioTek's PowerWave XS Absorbance Microplate Reader (Winooski, VT). Values were corrected for background OD from wells only containing medium.

\section{RESULTS AND DISCUSSION}

The ethyl acetate residue (10.7 g) of the ethanolic extract from E. illitum, afforded, after chromatographic 
procedures, compounds 1-5 (Figure 1). Their chemical structures were identified using spectroscopic techniques (FTIR, HRESIMS, and NMR) and by comparison with data reported in literature. Their found spectral data are shown below.

\section{Kumatakenin (1)}

Yellow powder; IR $\nu_{\max }: 3236,2948,2362,2337,1656$, $1600,1583,1497 \mathrm{~cm}^{-1}$; HRESIMS obsd. m/z 313.0663 [M $-\mathrm{H}]^{-}$calcd. for $\mathrm{C}_{17} \mathrm{H}_{14} \mathrm{O}_{6}, 313.0790 ;{ }^{1} \mathrm{H}$ NMR $(700 \mathrm{MHz}$, DMSO-d $)_{6} \delta=12.69(1 \mathrm{H}, \mathrm{s}, 5-\mathrm{OH}), 10.31(1 \mathrm{H}, \mathrm{br}$, 4'- $\mathrm{OH}), 7.99$ (2H, d, J = 8.9 Hz, H-2', H-6'), 6.97 (2H, d, $\left.J=8.6 \mathrm{~Hz}, \mathrm{H}-3^{\prime}, \mathrm{H}-5^{\prime}\right), 6.76(1 \mathrm{H}, \mathrm{d}, J=1.6 \mathrm{~Hz}, \mathrm{H}-8)$, $6.39(1 \mathrm{H}, \mathrm{d}, J=1.6 \mathrm{~Hz}, \mathrm{H}-6), 3.87(3 \mathrm{H}, \mathrm{s}, 7-\mathrm{OMe}), 3.81$ $(3 \mathrm{H}, \mathrm{s}, 3-\mathrm{OMe}) .{ }^{13} \mathrm{C}$ NMR $\left(176 \mathrm{MHz}, \mathrm{DMSO}-\mathrm{d}_{6}\right) \delta=178.5$ (C-4), 165.6 (C-7), 161.4 (C-5), 160.8 (C-4'), 156.8 (C-9), 156.4 (C-2), 138.3 (C-3), 130.7 (C-2', C-6'), 120.9 (C-1'), 116.1 (C-3', C-5'), 105.7 (C-10), 98.2 (C-6), 92.8 (C-8), 60.2 (3-OMe), 56.6 (7-OMe). The spectroscopic data $\left({ }^{1} \mathrm{H}\right.$ and ${ }^{13} \mathrm{C}$ NMR) were comparable with published values. ${ }^{7,8}$ Assignments were confirmed by HSQC and HMBC experiments.

\section{Ermanin (2)}

Yellow solid; IR $\nu_{\text {max }}: 3130,2936,2840,2361,2050,1650$, 1604, $1498 \mathrm{~cm}^{-1}$; HRESIMS obsd. m/z 313.0706 [M $\mathrm{H}]^{-}$calcd. for $\mathrm{C}_{17} \mathrm{H}_{14} \mathrm{O}, 313.0790 ;{ }^{1} \mathrm{H}$ NMR $(700 \mathrm{MHz}$, DMSO-d $) \delta=12.65(1 \mathrm{H}, \mathrm{s}, 5-\mathrm{OH}), 8.03(2 \mathrm{H}, \mathrm{d}, J=8.8$ Hz, H-2', H-6'), 7.14 (2H, d, J = 9.1 Hz, H-3', H-5'), 6.46 $(1 \mathrm{H}, \mathrm{d}, J=1.7, \mathrm{H}-8), 6.21(1 \mathrm{H}, \mathrm{d}, J=1.9 \mathrm{H}-6), 3.87(3 \mathrm{H}$, s, 4'-OMe), 3.80 (3H, s, 3-OMe). ${ }^{13} \mathrm{C}$ NMR $(176 \mathrm{MHz}$, DMSO-d $) \delta=178.0(\mathrm{C}-4), 164.4(\mathrm{C}-7), 161.4\left(\mathrm{C}-4^{\prime}\right)$, 161.3 (C-5), 156.5 (C-9), 155.2 (C-2), 138.0 (C-3), 130.0 (C-2', C-6’), 122.2 (C-1'), 114.3 (C-3', C-5'), 104.2 (C-10), 98.7 (C-6), 93.9 (C-8), 59.8 (3-OMe), 55.5 (4'-OMe). The spectroscopic data $\left({ }^{1} \mathrm{H}\right.$ and $\left.{ }^{13} \mathrm{C} \mathrm{NMR}\right)$ were comparable with published values. ${ }^{9}, 10$ Assignments were confirmed by HSQC and HMBC experiments.

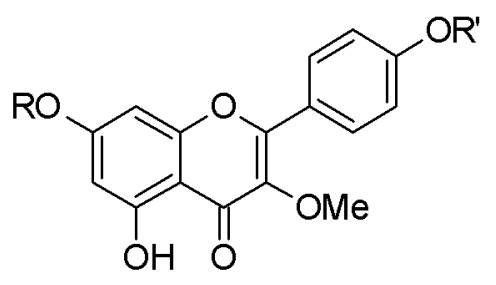

$$
1 \mathrm{R}=\mathrm{CH}_{3}, \mathrm{R}^{\prime}=\mathrm{H}
$$

$$
2 \mathrm{R}=\mathrm{H}, \mathrm{R}^{\prime}=\mathrm{CH}_{3}
$$

\section{7-Methoxy-aromadendrin (3)}

Yellow solid; IR $\nu_{\max }: 3144,2939,2258,1716,1636$, 1616, 1595, 1573, 1501, $1443 \mathrm{~cm}^{-1}$; HRESIMS obsd. m/z $301.0717[\mathrm{M}-\mathrm{H}]^{-}$calcd. for $\mathrm{C}_{16} \mathrm{H}_{14} \mathrm{O}_{6}, 301.0790 ;{ }^{1} \mathrm{H} \mathrm{NMR}$ $\left(700 \mathrm{MHz}, \mathrm{DMSO}-\mathrm{d}_{\sigma}\right) \delta=11.88(1 \mathrm{H}, \mathrm{s}, 5-\mathrm{OH}), 9.57(1 \mathrm{H}, \mathrm{s}$, 4'-OH), 7.33 (2H, d, J = $8.2 \mathrm{~Hz}, \mathrm{H}-2$ ', H-6'), 6.80 (2H, d, J $\left.=8.5 \mathrm{~Hz}, \mathrm{H}-3^{\prime}, \mathrm{H}-5^{\prime}\right), 6.12(1 \mathrm{H}, \mathrm{d}, J=2.1 \mathrm{~Hz}, \mathrm{H}-6), 6.10$ $(1 \mathrm{H}, \mathrm{d}, J=1.9 \mathrm{~Hz}, \mathrm{H}-8), 5.82(1 \mathrm{H}, \mathrm{d}, J=6.4 \mathrm{~Hz}, 3-\mathrm{OH})$, $5.11(1 \mathrm{H}, \mathrm{d}, J=11.3, \mathrm{H}-2), 4.66(1 \mathrm{H}, \mathrm{dd}, J=11.5,6.3 \mathrm{~Hz}$, H-3), 3.79 (3H, s, 7-OMe); ${ }^{13} \mathrm{C}$ NMR (176MHz, DMSO-d $)$ $\delta=199.0$ (C-4), 168.0 (C-7), 163.4 (C-5), 163.0 (C-9), 158.2 (C-4'), 130.0 (C-2', C-6'), 127.9 (C-1'), 115.4 (C-3', C-5'), 101.8 (C-10), 95.4 (C-6), 94.3 (C-8), 83.5 (C-2), 72.0 (C-3), 56.4 (7-OMe). The spectroscopic data $\left({ }^{1} \mathrm{H}\right.$ and ${ }^{13} \mathrm{C}$ NMR $)$ were comparable with published values. ${ }^{11}$ Assignments were confirmed by HSQC and HMBC experiments.

\section{Naringenin (4)}

Yellow solid; IR $\nu_{\max }: 3115,3055,2019,2819,2831,2700$, 2349, 2286, 2051, 1898, 1627, 1600, 1586, 1519, 1496 $\mathrm{cm}^{-1}$; HRESIMS obsd. $\mathrm{m} / \mathrm{z} 271.0603[\mathrm{M}-\mathrm{H}]^{-}$calcd. for $\mathrm{C}_{15} \mathrm{H}_{12} \mathrm{O}_{5}, 271.0685 ;{ }^{1} \mathrm{H}$ NMR $(700 \mathrm{MHz}$, DMSO-d $) \delta=$ $12.16(1 \mathrm{H}, \mathrm{s}, 5-\mathrm{OH}), 10.80(1 \mathrm{H}, \mathrm{br}$ s, $7-\mathrm{OH}), 9.59(1 \mathrm{H}$, br s, 4'- $-\mathrm{OH}), 7.32\left(2 \mathrm{H}, \mathrm{d}, J=8.5 \mathrm{~Hz}, \mathrm{H}-2^{\prime}, \mathrm{H}^{-} 6^{\prime}\right), 6.80(2 \mathrm{H}$, d, $J=8.3 \mathrm{~Hz}, \mathrm{H}-3^{\prime}, \mathrm{H}^{-5}$ '), 5.88 (2H, s, H-6, H-8), 5.45 (1H, $\mathrm{dd}, J=12.8,2.8 \mathrm{~Hz}, \mathrm{H}-2), 3.28(1 \mathrm{H}, \mathrm{dd}, J=17.0,12.9$, $\mathrm{H}-3$ trans.), 2.69 (1H, dd, $J=17.1,2.9 \mathrm{~Hz}, \mathrm{H}-3$ cis.); ${ }^{13} \mathrm{C}$ NMR (176MHz, DMSO-d $) \delta=196.8(\mathrm{C}-4), 167.2(\mathrm{C}-7)$, 163.9 (C-5), 163.4 (C-9), 158.2 (C-4'), 129.3 (C-1'), 128.8 (C-2', C-6'), 115.6 (C-3', C-5'), 102.2 (C-10), 96.3 (C-6), 95.5 (C-8), 78.9 (C-2), 42.4 (C-3). The spectroscopic data $\left({ }^{1} \mathrm{H}\right.$ and ${ }^{13} \mathrm{C}$ NMR) were comparable with published values. ${ }^{12,13}$

\section{4-Hydroxybenzoic acid (5)}

White solid; IR $\nu_{\max }: 3400,2255,2128,1654,1049,1023$, 999, 824, 762, 625, $614 \mathrm{~cm}^{-1}$; HRESIMS obsd. $\mathrm{m} / \mathrm{z} 137.0242$ $[\mathrm{M}-\mathrm{H}]^{-}$calcd. for $\mathrm{C}_{7} \mathrm{H}_{6} \mathrm{O}_{3}, 137.0317 ;{ }^{1} \mathrm{H}$ NMR $(700 \mathrm{MHz}$,

Figure 1: Structures of compounds 1-5 isolated from the aerial parts of Eupatorium illitum 


\begin{tabular}{|c|c|c|c|c|c|}
\hline Compound & A549 & HBL-100 & SW1573 & HeLa & T-47D \\
\hline 1 & 163 & 12 & 5 & 10 & 250 \\
\hline 2 & 16 & 11 & 7 & 8 & 58 \\
\hline 4 & 40 & 22 & 10 & 7 & 49 \\
\hline 5 & 12 & 10 & 8 & 7 & 13 \\
\hline
\end{tabular}

DMSO-d $) \delta=7.79(2 \mathrm{H}, \mathrm{d}, J=8.5 \mathrm{~Hz}, \mathrm{H}-2, \mathrm{H}-6), 6.82(2 \mathrm{H}$, $\mathrm{d}, J=8.7 \mathrm{~Hz}, \mathrm{H}-3, \mathrm{H}-5) ;{ }^{13} \mathrm{C}$ NMR $(176 \mathrm{MHz}$, DMSO-d $)$ $\delta=167.6(\mathrm{COOH}), 162.0(\mathrm{C}-4), 132.0(\mathrm{C}-2, \mathrm{C}-6), 121.8$ (C-1), 115.6 (C-3, C-5). The spectroscopic data $\left({ }^{1} \mathrm{H}\right.$ and ${ }^{13} \mathrm{C}$ NMR) were comparable with published values. ${ }^{14}$

\section{Antiproliferative activity}

Selected isolates were evaluated for their antiproliferative activity against the human solid tumor cell lines A549 (lung), HBL-100 (breast), HeLa (cervix), SW1573 (lung), and T-47D (breast). The effect of these compounds on the aforementioned cell lines, expressed as the value $\mathrm{GI}_{50}$ (concentration causing $50 \%$ of growth inhibition), is presented in Table 1.

\section{CONCLUSION}

In summary, we have reported the isolation of four flavonoids from Eupatorium illitum in addition to 4-Hydroxybenzoic acid. All compounds are reported for first time in this plant. The study of the antiproliferative activity against human solid tumor cell lines showed that the compounds are able to inhibit cell growth in all cell lines tested.

\section{CONFLICT OF INTEREST}

Authors do not have any conflict of interest.

\section{ACKNOWLEDGMENTS}

This project has been supported by research grant FONDOCYT 2009-16, Ministerio de Educación Superior, Ciencia y Tecnología (MESCYT), Dominican Republic. QAC thanks Dr. Mehdi Keramane (Mc Master University, Canada) for a diligent technical assistance. JMP thanks the EU Research Potential (FP7-REGPOT-2012-CT201231637-IMBRAIN), the European Regional Development Fund (FEDER), and the Spanish Instituto de Salud Carlos
III (PI11/00840) for financial support. GBP thanks Fundación Caja Canarias for a postgraduate grant.

\section{REFERENCES}

1. Liogier AH. La Flora de la Española VIII. $1^{\text {st }}$ ed. San Pedro de Macorís: Universidad Central del Este; 1996.

2. Zhang ML, Wu M, Zhang JJ, Irwin D, Gu YC, Shi QW. Chemical Constituents of Plants from the Genus Eupatorium. Chem. Biodivers. 2008; 5(1): 40-55.

3. Kupchan SM, Sigel CW, Knox JR, Udayamurthy MS. 1969. Tumor inhibitors. XXXVI. Eupatin and eupatoretin, two cytotoxic flavonols from Eupatorium semiserratum. J. Org. Chem. 1969; 34(5): 1460-3.

4. Militão GCG, Albuquerque MRJR, Pessoa ODL, Pessoa C, Moraes MEA, De Moraes MO, Costa-Lotufo LV. Cytotoxic activity of nepetin, a flavonoid from Eupatorium ballotaefolium HBK. Pharmazie 2004; 59(12): 965-6.

5. Herrerias T, Oliveira AA, Belem ML, Oliveira BH, Carnieri EGS, Cadena SMSC, Noleto GR, et al. Effects of natural flavones on membrane properties and cytotoxicity of HeLa cells. Rev. Bras. Farmacogn. 2010; 20(3): 403-8.

6. Miranda PO, Padrón JM, Padrón JI, Villar J, Martín VS. Prins-Type Synthesis and SAR Study of Cytotoxic Alkyl Chloro Dihydropyrans. Chem Med Chem. 2006; 1(3): 323-9.

7. Zhang X, Li B, Zhou M, Yuan X, Zhang G. Chemical Constituents of Buddleja brachystachya Diels. Chin. J. Appl. Environ. Biol. 2006; 12(3): 338-41.

8. Villaflores OB, Macabeo APG, Gehle D, Krohn K, Franzblau SG, Aguinaldo AM. Phytoconstituents from Alpinia purpurata and their in vitro inhibitory activity against Mycobacterium tuberculosis. Phcog. Mag. 2010; 6(24): 339-44.

9. Calvert DJ, Cambie RC, Davis BR. ${ }^{13} \mathrm{C}$ NMR Spectra of Polymethoxy- and Methylenedioxyflavonols. Org. Magn. Reson. 1979; 12(10): 583-6.

10. Kim BG, Kim H, Kim JH, Lim Y, Ahn JH. Synthesis of Ermanin, 5,7-Dihydroxy-3,4'-dimethoxyflavone from Kaempferol, 3,5,7,4'-Tetrahydroxyflavone with Two O-Methyltransferases Expressed in E. coli. Bull. Korean Chem. Soc. 2006; 27(3): 357-8.

11. Chiappini I, Fardella G, Menghini A, Rossi C. Flavonoids from Dittrichia viscosa. Planta Med. 1982; 44(3): 159-61.

12. Ibrahim ARS, Galal AM, Ahmed MS, Mossa GS. O-Demethylation and Sulfation of 7-Methoxylated Flavanones by Cunninghamella elegans. Chem. Pharm. Bull. 2003; 51(2): 203-6.

13. Kim DH, Kim BG, Lee Y, Ryu JY, Lim Y, Hur HG, Ahn JH. Regiospecific methylation of Naringenin to ponciretin by soybean O-methyltransferase expressed in Escherichia coli. Journal of Biotechnology 2005; 119(2): 155-62.

14. Cho JY, Moon JH, Seong DY, Park KH. Antimicrobial Activity of 4-Hydroxybenzoic Acid and trans 4-Hydroxycinnamic Acid Isolated and Identified from Rice Hull. Biosci. Biotechnol. Biochem. 1998; 62(11): 2273-6. 